\title{
Implementación de la modulación por densidad de pulsos PDM en un sistema de generación de ozono y su efecto en la curva característica de eficiencia
}

\author{
William Andrés Cifuentes Rojas* \\ Diego Fernando Echeverry lbarra** \\ Hernando Vásquez Palacios ${ }^{* * *}$
}

Recibido: 04/03/2013 - Aceptado: 27/06/2014

\begin{abstract}
Resumen
Los sistemas de generación de ozono generalmente utilizan la modulación por amplitud de pulsos (PAM) para variar o controlar la concentración de ozono. En este trabajo se presentan los resultados obtenidos luego de la implementación de la modulación por densidad de pulsos (PDM) en la fuente de potencia de un sistema de generación de ozono y el mejoramiento logrado en la curva característica de la eficiencia. Las técnicas PAM y PDM se aplicaron en un sistema de generación de ozono con inversor push-pull auto-resonante. La amplitud de voltaje ajustada en la onda de potencia PDM se determinó de la condición de la onda PAM donde se obtuvo la máxima eficiencia en el $60 \%$ de los puntos de operación del sistema de generación de ozono.
\end{abstract}

Palabras clave: generador de ozono, eficiencia, modulación, DBD, PAM, PDM, DSP, PUSH-PULL

\footnotetext{
Estudiante de maestría con énfasis en automática - Grupo de Investigación en Alta Tensión GRALTA- Universidad del Valle, dirección: Calle 13 No 100-00 Ciudad Universitaria Meléndez edif 356 Cali-Colombia, Teléfonos: +57 (2) 3321948/3334252 ext 113, correo electrónico: william.cifuentes@correounivalle.edu.co.

* PhD, Profesor asociado - Grupo de Investigación en Alta Tensión GRALTA - Universidad del Valle, dirección: Calle 13 N ${ }^{\circ}$ 100-00 Ciudad Universitaria Meléndez edif. 356 Cali-Colombia, Teléfonos: +57 (2) 3321948/3334252/3212478 ext. 113, correo electrónico: diego.echeverry@correounivalle.edu.co, Fax: +57 (2) 3212151

*** MSc, Profesor titular - Grupo de Investigación en Control Industrial GICI -Universidad del Valle, dirección: Calle 13 N. ${ }^{\circ}$ 100-00 Ciudad Universitaria Meléndez edif. 353 Cali-Colombia, Teléfonos: +57 (2) 3391780/3212100 ext.2108, correo electrónico: hernando.vasquez@correounivalle.edu.co
} 


\title{
Implementation of pulse-density modulation PDM in an ozone generation system and its contribution to the characteristic efficiency curve
}

\begin{abstract}
The ozone generation systems generally use the pulse amplitude modulation (PAM) to vary or control the ozone concentration. The results obtained after the implementation of pulse density modulation (PDM) in the power supply system of an ozone generation and the improvement achieved in the efficiency curve is presented in this paper. PAM and PDM techniques were applied in ozone generation system with push-pull inverter resonant self. The adjusted voltage amplitude of the PDM waveform power is determined from the condition of the PAM waveform where the maximum efficiency was obtained; Thus achieving increased efficiency in $60 \%$ of operating points ozone generation system.
\end{abstract}

Key words: Ozone generation, efficiency, modulation, DBD, PAM, PDM, DSP, PUSH-PULL. 


\section{INTRODUCCIÓN}

Los sistemas de generación de ozono son utilizados en múltiples procesos industriales, entre los cuales se destacan la desinfección y la potabilización de agua [1]. Otra aplicación del ozono es el pre-tratamiento de desechos contaminantes como, por ejemplo, las vinazas las cuales provienen de los procesos de destilación de alcohol; el pre-tratamiento consiste en la aplicación de ozono en fase gaseosa mediante burbujeo, lográndose una reducción de la demanda química de oxígeno (DQO) de la vinaza cruda [2].

En la práctica es común que en un mismo sistema de generación de ozono se requiera variar la concentración en un rango determinado para atender las necesidades del proceso [3] y, a la vez, garantizar que la eficiencia del sistema sea lo más alta posible, con el fin de contribuir a la sostenibilidad económica y a la gestión energética del proceso. En las celdas de descarga de barrera dieléctrica (DBD) se logra obtener una alta eficiencia en la síntesis de ozono cuando la intensidad de campo eléctrico reducido dentro de la celda se acerca a un valor óptimo; el campo eléctrico reducido óptimo depende de la amplitud del voltaje aplicado [4] al cual llamaremos voltaje óptimo.

Por el contrario, para amplitudes de voltaje menores al óptimo la eficiencia empieza a decaer hasta llegar a valores muy bajos. Esta es la razón por la cual se presentan bajas eficiencias cuando se aplican bajos voltajes para generar pequeñas concentraciones de ozono en un equipo determinado.

Para variar y controlar la concentración de ozono, es necesario regular la potencia de descarga en el ozonizador, la cual es controlada por la fuente eléctrica del sistema [5]. Existen diferentes métodos de control de potencia utilizados en los sistemas para generar plasmas a presión atmosférica, como lo son, entre otros, PWM, PDM, PFM, PAM, [6].

La modulación PAM (Pulse Amplitude Modulation) consiste en el ajuste de la potencia mediante la variación de la amplitud de los pulsos de voltaje aplicados a la celda DBD manteniendo la frecuencia constante (Ver figura 1A). La modulación PDM (Pulse Density Modulation) consiste en el ajuste de la potencia mediante la sucesión de pulsos de alta frecuencia durante un tiempo TON y con pulsos atenuados en amplitud durante un tiempo TOFF. El período de tiempo de la onda PDM es constante y corresponde a la suma de los tiempos TON y TOFF (Ver figura 1B). La potencia varía conforme a la relación del ciclo de trabajo $\mathrm{D}=\mathrm{TON} /(\mathrm{TON}+\mathrm{TOFF})$.

Para el cálculo de la eficiencia de un generador de ozono es necesario medir la concentración de ozono, el flujo del gas y la potencia consumida, tal como se muestra en la Ecuación 1. Eficiencia expresada en forma paramétrica. Dependiendo del sistema de generación de ozono y de la formas de ondas de las señales eléctricas presentes, es necesario utilizar una determinada técnica para la medición de potencia. 


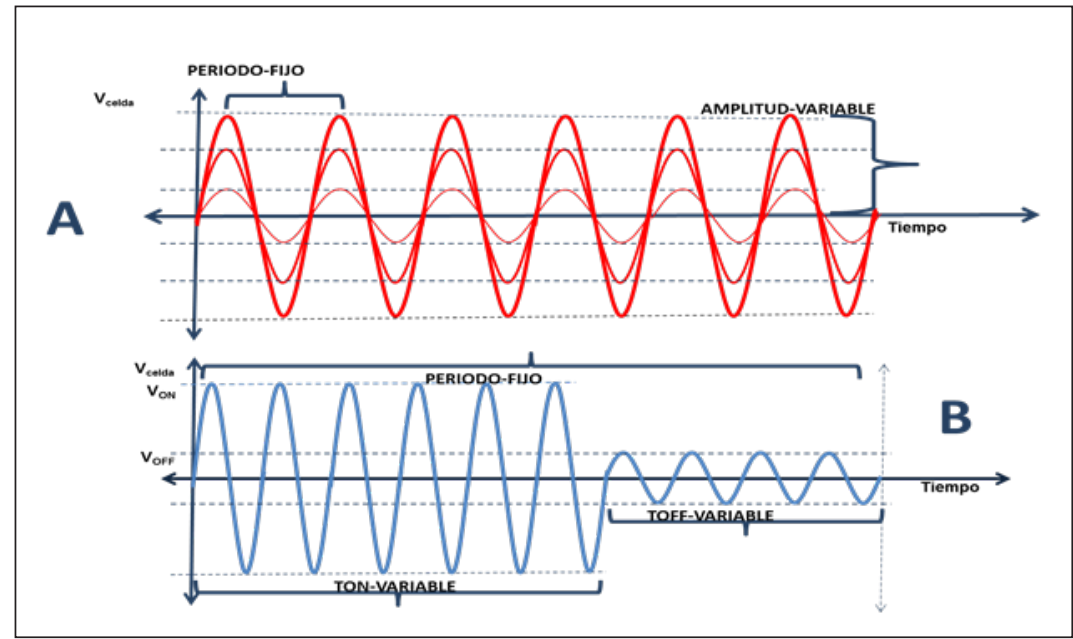

Figura 1. Formas de onda para la modulación PAM y PDM

Fuente: elaboración propia

Ecuación 1. Eficiencia expresada en forma paramétrica

$$
\eta O_{3}=60 \frac{\left[O_{3}\right] \times F}{P} \quad\left(\mathrm{~g} \cdot \mathrm{kWh}^{-1}\right)
$$

Donde: $\left[\mathrm{O}_{3}\right]$ es la concentración de ozono $\left(\mathrm{g} \cdot \mathrm{m}^{-3}\right), \mathrm{F}$ es el flujo de gas $\left(\mathrm{L} \cdot \mathrm{min}^{-1}\right), \mathrm{P}$ potencia consumida $(\mathrm{W})$.

Una metodología para incrementar la eficiencia del sistema de generación de ozono, considera el mejoramiento de la eficiencia de, al menos, uno de los dos bloques principales: la fuente de alto voltaje o la celda DBD. Entre las alternativas para mejorar la eficiencia de la fuente se pueden citar los pulsos de corta duración [7-10]. Sin embargo, actualmente se tienen grandes limitantes técnicas en la implementación de la fuente para generar estos pulsos; también se ha experimentado con el aumento de la frecuencia y la operación bajo resonancia [11-13]. Desde el punto de vista constructivo de las celdas, las investigaciones se han centrado principalmente en buscar el mejor punto de operación de una celda de descarga volumétrica [7, 14-17] y también se han evaluado diferentes materiales dieléctricos, dimensiones de las celdas VD y temperatura de refrigeración [18].

En este trabajo se demuestra que es posible aumentar la eficiencia en los puntos de operación de baja concentración de ozono al alimentar la celda DBD con una señal de alta frecuencia tipo PDM, ajustada a una amplitud óptima. 


\section{IMPLEMENTACIÓN DE UNA FUENTE ELÉCTRICA EXPERIMENTAL}

La fuente eléctrica implementada en este trabajo, que se muestra en la figura 2, permitió aplicar las diferentes técnicas de modulación de potencia estudiadas. Consta principalmente de una fuente primaria de DC, un convertidor DC-DC, y un inversor en resonancia

El convertidor DC-DC se implementó con una topología tipo buck la cual realiza el control de corriente DC que es aplicada al inversor, y por consiguiente, la corriente, suministrada a la carga; está compuesto por un mosfet M3, un diodo de conmutación rápida $\mathrm{D} 5$ y un inductor $\mathrm{L} 1$ el cual se comparte con el inversor. El inversor es alimentado con corriente y se implementó con una configuración push-pull con transformador, que se autoajusta a la frecuencia de resonancia, paralelo del circuito formado por el transformador elevador T1 y la celda de descarga DBD. El control se implementó mediante el circuito UC3872 de Texas Instrument [3, 19, 20]. El hardware del controlador está basado en un DSP TMS320F2812 de texas instrument, el cual ejecuta el algoritmo que controla el convertidor buck.

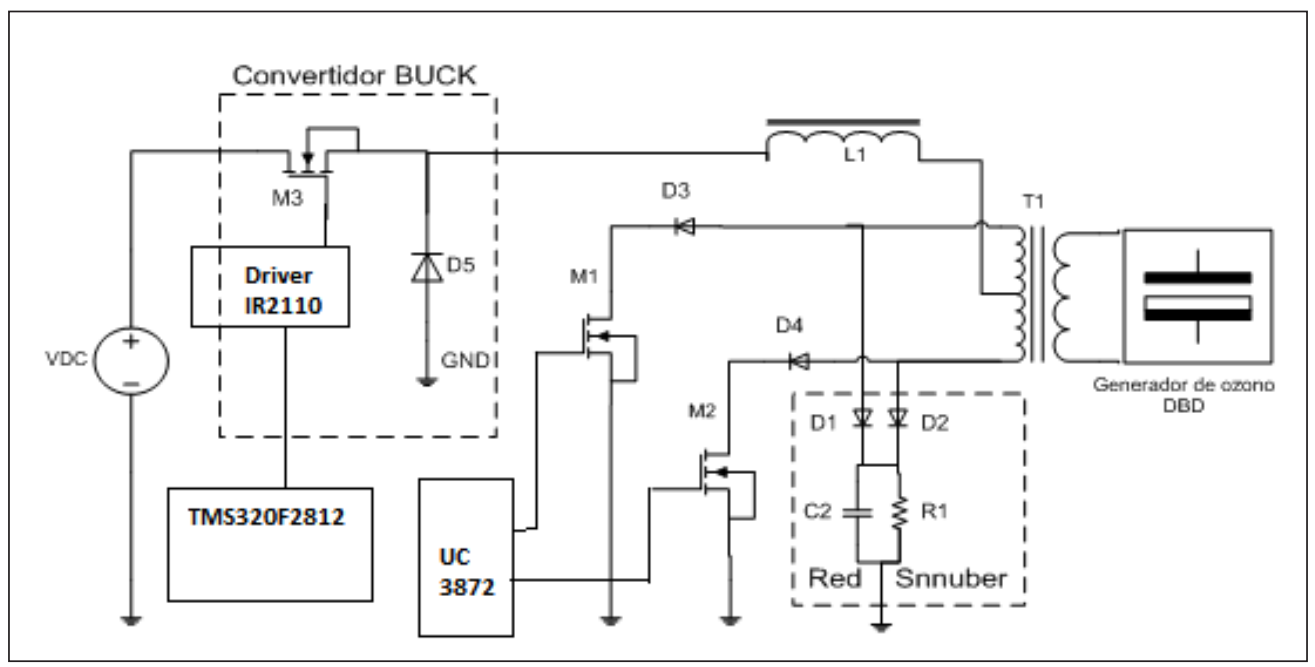

Figura 2. Fuente experimental implementada

Fuente: elaboración propia

\section{METODOLOGÍA}

En primer lugar se plantea la realización del montaje experimental y la instrumentación necesaria. En la tabla 1 se relacionan las variables, las unidades de medida y los equipos de medición utilizados en el experimento. 
Tabla 1 Variables medidas y equipos de medición

\begin{tabular}{|l|c|l|}
\hline Variable & Unidad & Equipo de medición \\
\hline Voltajes y Corrientes de DC & $\mathrm{V}, \mathrm{A}$ & Osciloscopio Gwinstek 2000 \\
\hline Frecuencia de AC & $\mathrm{Hz}$. & Fluke 196B scopemeter \\
\hline Voltajes de AC en alta tensión & $\mathrm{V}$ & Tektronix P601SA, Fluke 196B \\
\hline Concentración de ozono & $\mathrm{g} / \mathrm{m} 3$ & Analizador IN-USA Mini HiCom \\
\hline Flujo de oxígeno u ozono & $\mathrm{cm} 3 / \mathrm{min}$ & Rotametro dwyer \\
\hline
\end{tabular}

Fuente: elaboración propia

El montaje neumático se detalla en la Figura 3 Esquema de bloques e instrumentación para la medición de concentración de ozono y comprende una fuente de oxígeno, un regulador de presión, un medidor de flujo, una válvula de ajuste, un generador y un analizador de ozono. El flujo de entrada de $\mathrm{O}_{2}$, al cual se aplicaron los experimentos, es de $1 \mathrm{~L} / \mathrm{min}$ y se asume que el mismo flujo se presenta a la salida del generador.

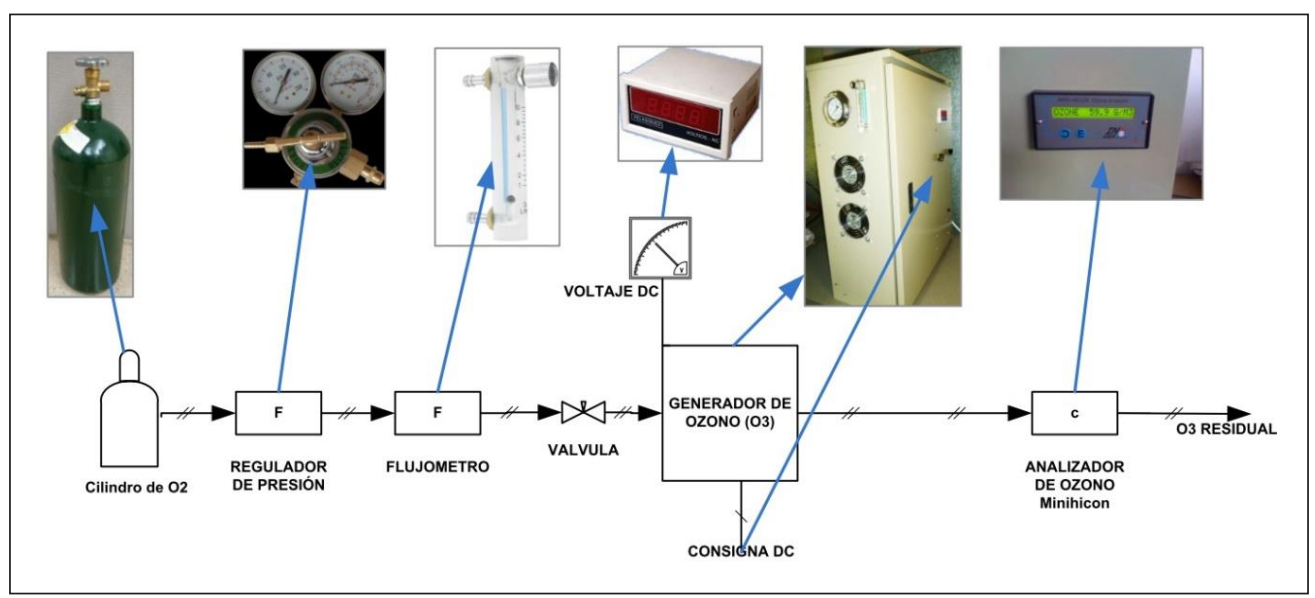

Figura 3. Esquema de bloques e instrumentación para la medición de concentración de ozono Fuente: elaboración propia

En la Figura 4 Esquema eléctrico del sistema y la instrumentación para medir potencia. se muestra el diagrama de la fuente eléctrica modulada del sistema de generación de ozono y la instrumentación necesaria para la medición de potencia. En el diagrama se detallan los puntos en los cuales se hizo el cálculo de la eficiencia: la entrada del inversor resonante, la cual se nombrará como eficiencia $1\left(\eta_{1}\right)$, y en la celda DBD, la cual se nombrará como eficiencia $2\left(\eta_{2}\right)$. 


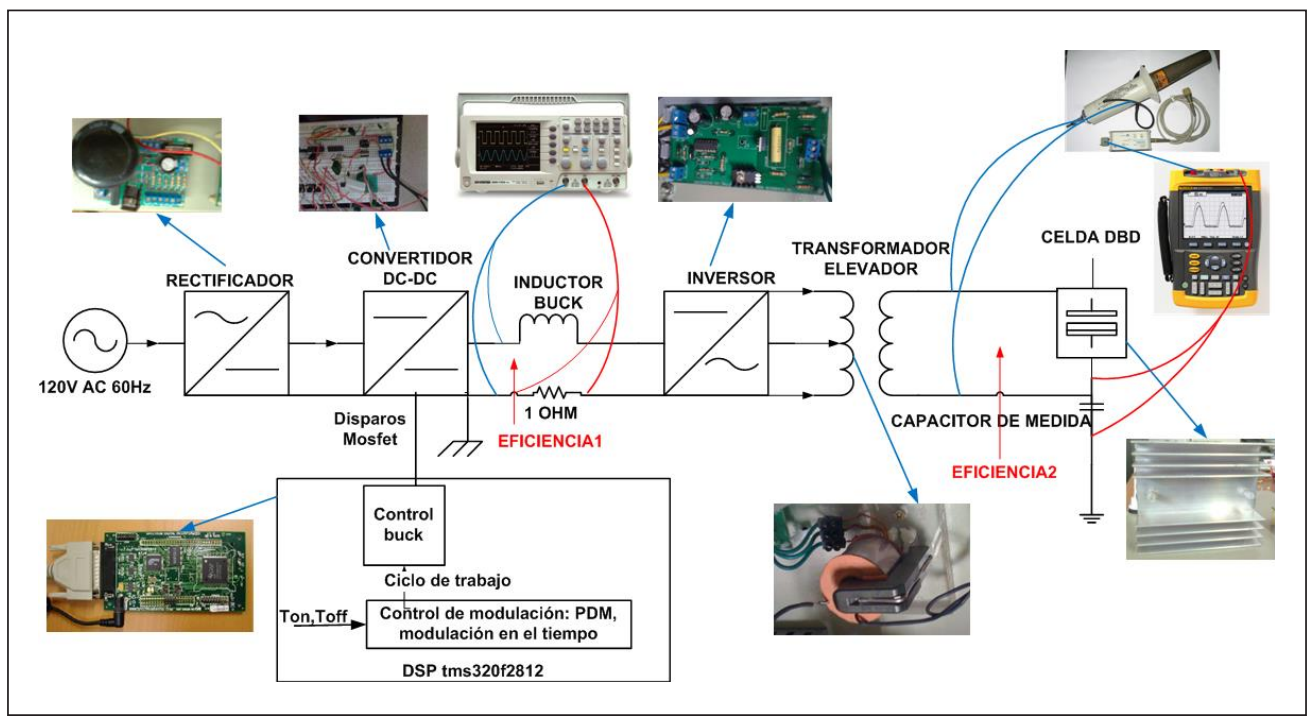

Figura 4. Esquema eléctrico del sistema y la instrumentación para medir potencia.

Fuente: elaboración propia

Primero se obtuvo la curva de eficiencia del sistema de generación de ozono, provocada por la variación de la amplitud de la onda de voltaje (PAM), para un flujo de $1 \mathrm{~L} / \mathrm{min}$ de $\mathrm{O}_{2}$, variando de forma ascendente el voltaje DC de entrada $\left(\mathrm{V}_{\mathrm{DC}}\right)$ en pasos de $0.5 \mathrm{~V}$ entre el valor en el cual se produce la mínima concentración detectable y la máxima concentración. Para este caso particular se realizó la variación entre $53 \mathrm{~V}$ y $60 \mathrm{~V}$.

Simultáneamente con el barrido de DC, se realiza la medición de frecuencia $\left(\mathrm{F}_{\mathrm{AC}}\right)$, concentración de $\mathrm{O}_{3}\left(\left[\mathrm{O}_{3}\right)\right.$ y flujo de $\mathrm{O}_{2}$; además, se capturan y almacenan las siguientes variables: voltaje $\left(\mathrm{V}_{\mathrm{DC}}\right)$ y corriente $\left(\mathrm{I}_{\mathrm{DC}}\right)$ en la salida del convertidor $\mathrm{DC} /$ $\mathrm{DC}$, voltaje $\left(\mathrm{V}_{\mathrm{AC}}\right)$ en el secundario del transformador y voltaje $\left(\mathrm{V}_{\mathrm{QAC}}\right)$ en el capacitor de medición de carga eléctrica. La eficiencia se estimó en dos puntos del sistema, uno desde la salida del convertidor DC/DC ( $\eta 1)$ y otro a la entrada de la celda de descarga

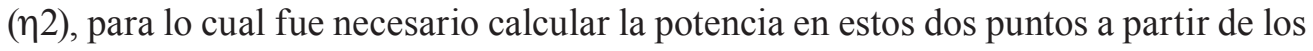
datos almacenados de corriente y voltaje. De este modo se obtienen las curvas de concentración de $\mathrm{O}_{3}$ frente a la eficiencia $\eta_{1}$ y $\eta_{2}$ a partir de las cuales se identifica el punto de máxima eficiencia y su correspondiente valor de voltaje de DC, que fue de 57,5V.

Una vez se encuentra el voltaje que origina la máxima eficiencia bajo PAM, se plantea la realización del experimento aplicando la técnica de modulación de potencia PDM. Inicialmente se ajusta el voltaje de entrada al convertidor buck el cual corresponde al voltaje del punto de máxima eficiencia hallado anteriormente. Luego se hace un barrido entre el $5 \%$ y el $95 \%$ del ciclo de trabajo bajo modulación PDM, variando 
la relación de $\mathrm{t}_{\mathrm{ON}} / \mathrm{t}_{\mathrm{OFF}}$ en escalones de $10 \%$, a una frecuencia de modulación PDM fija de $154 \mathrm{~Hz}$.

Para este experimento, se realiza la medición de las siguientes variables: frecuencia AC, concentración de $\mathrm{O}_{3}$, flujo de $\mathrm{O}_{2}$, voltaje de $\mathrm{AC}$ en el secundario del transformador, voltaje en el capacitor de medición de carga eléctrica, voltaje y corriente de DC a la entrada del inversor, y luego se calculan las curvas de concentración de $\mathrm{O}_{3}$ frente a la eficiencia $\eta_{1}$ y $\eta_{2}$ para las modulaciones aplicadas:

\section{RESULTADOS}

En la metodología experimental se realizó la caracterización estática del sistema bajo la modulación de potencia PAM, con el fin de obtener la curva de eficiencia descrita por el sistema e identificar el rango de condiciones de operación óptimas. En la Figura 5 Relación entre la $\eta 1$ y el voltaje de DC con modulación PAM se muestra la relación entre la eficiencia $\eta 1$ a la entrada del inversor y el voltaje de DC cuando se utiliza modulación PAM. Se observa que ante un aumento del voltaje de DC la $\eta 1$ también se incrementa y dicho comportamiento se mantiene hasta el punto máximo en $40 \mathrm{gO}_{3} /$ $\mathrm{kWh}$ para un voltaje DC de 56,4V. Finalmente la eficiencia permanece constante en un rango de máxima eficiencia hasta $58,2 \mathrm{~V}$, punto a partir del cual la eficiencia decae hasta $32 \mathrm{~g} / \mathrm{kWh}$ con un voltaje de $61 \mathrm{~V}$ al final del rango evaluado.

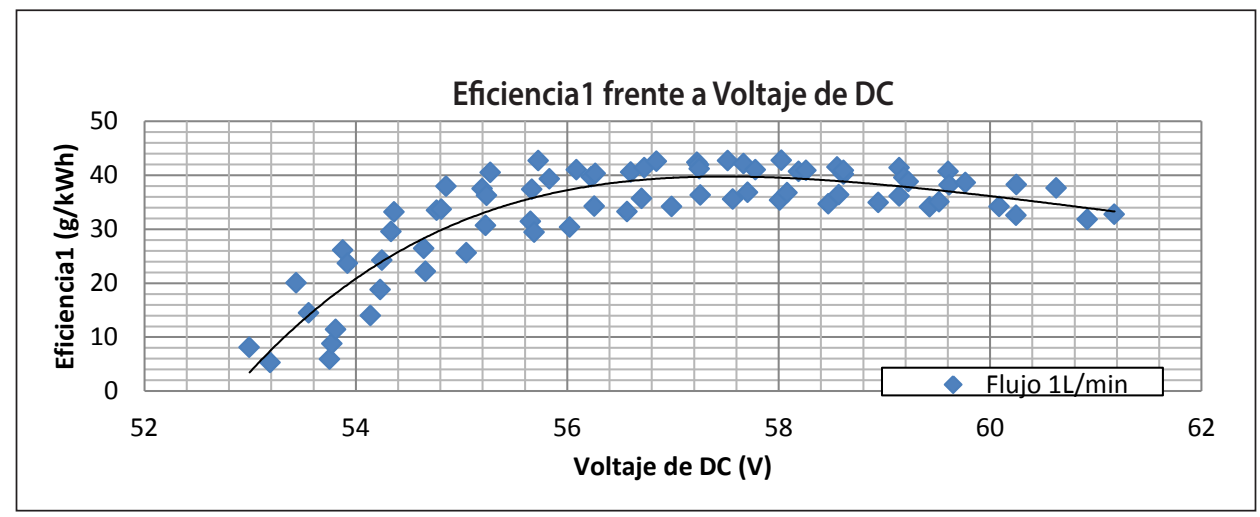

Figura 5. Relación entre la ๆ1 y el voltaje de DC con modulación PAM

Fuente: elaboración propia

En la Figura 6 Relación entre la $\rceil 2$ y el voltaje de DC con modulación PAM se muestra la relación entre la $\eta 2$ a la salida del inversor y el voltaje de DC. Se observa que ante un aumento del voltaje de DC, la $\rceil 2$ también se incrementa, y dicho com-

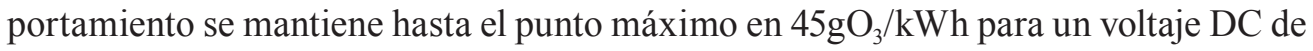
$56 \mathrm{~V}$. Finalmente la eficiencia permanece constante en un rango de máxima eficiencia 
hasta $57,6 \mathrm{~V}$; a partir de este punto la eficiencia decae hasta $36 \mathrm{~g} / \mathrm{kWh}$ con un voltaje de $61 \mathrm{~V}$ al final del rango evaluado.

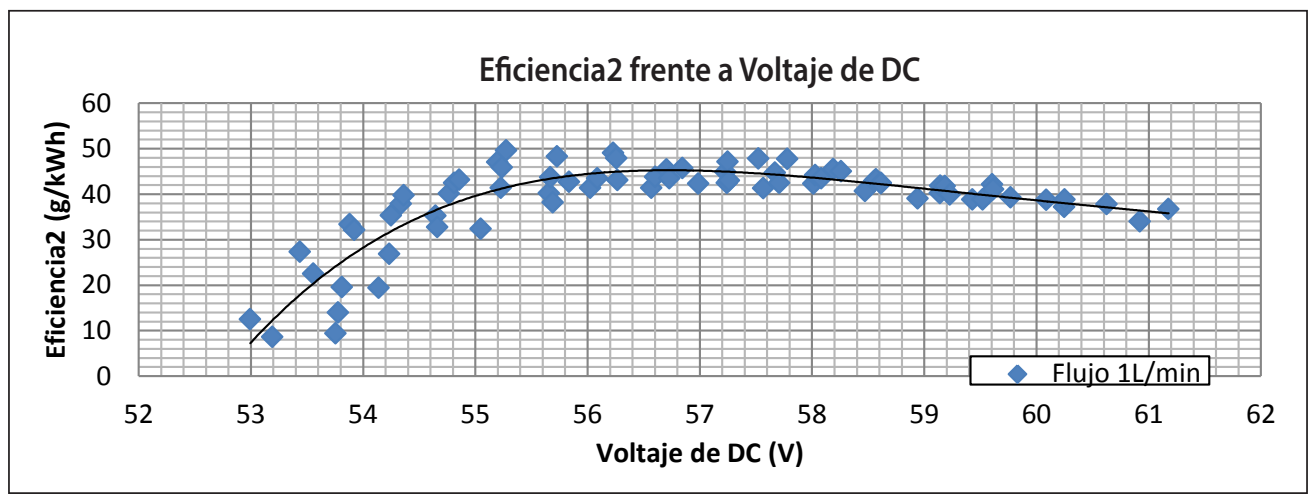

Figura 6. Relación entre la n2 y el voltaje de DC con modulación PAM

Fuente: elaboración propia

De las curvas anteriores se observa que en los rangos de alta eficiencia el valor intermedio de DC es de 57,5V, amplitud en la cual se fijan los pulsos para evaluar la técnica de modulación PDM.

Para el caso de las dos técnicas de modulación estudiadas, en las gráficas siguientes se muestran las curvas de la eficiencia en función de la concentración de ozono. Estas curvas permiten comparar el desempeño que presentan las modulaciones en el rango de trabajo del generador. En la Figura 7 Eficiencia $\eta 1$ frente a concentración de $\mathrm{O} 3$ para la modulación PAM y PDM se presentan los resultados del desempeño del sistema bajo la modulación PAM y PDM, en un rango de puntos de concentración entre 0 y $19.5 \mathrm{~g} /$ $\mathrm{m}^{3}$. En la curva se observa para el caso de PAM, que la $\eta 1$ mejora conforme aumenta la concentración de ozono, desde cero hasta el punto de máxima eficiencia el cual corresponde a 38g/kWh@13.5g/ $\mathrm{m}^{3}$, luego permanece constante hasta alcanzar valores de concentración de $15 \mathrm{~g} / \mathrm{m}^{3}$; para concentraciones mayores la eficiencia comienza a decaer.

La curva para PDM muestra que la $\eta 1$ aumenta más rápidamente conforme aumenta la concentración y alcanza un valor de eficiencia alto $40 \mathrm{~g} / \mathrm{kWh} @ 4 \mathrm{~g} / \mathrm{m}^{3}$; luego se alcanza una máxima eficiencia de $45 \mathrm{~g} / \mathrm{kWh} @ 10 \mathrm{~g} / \mathrm{m}^{3}$. Por otro lado, en la Figura 8 Eficiencia $\eta 2$ frente a concentración de $\mathrm{O} 3$ para la modulación PAM y PDM se observa que la $\eta 2$ para el caso de PAM aumenta desde el origen hasta obtener la eficiencia máxima en $44 \mathrm{~g} / \mathrm{kWh} @ 12 \mathrm{~g} / \mathrm{m}^{3}$, luego permanece constante hasta el punto de trabajo $14 \mathrm{~g} / \mathrm{m}^{3}$, a partir del cual comienza a decaer hasta $40 \mathrm{~g} / \mathrm{kWh} @ 19.5 \mathrm{~g} / \mathrm{m}^{3}$. Para el caso de PDM se observa que la $\eta_{2}$ alcanza un valor de eficiencia alto de $50 \mathrm{~g} / \mathrm{kWh} @ 4 \mathrm{~g} / \mathrm{m}^{3}$ y luego la máxima eficiencia se presenta a $56 \mathrm{~g} / \mathrm{kWh} @ 9 \mathrm{~g} / \mathrm{m}^{3}$. 


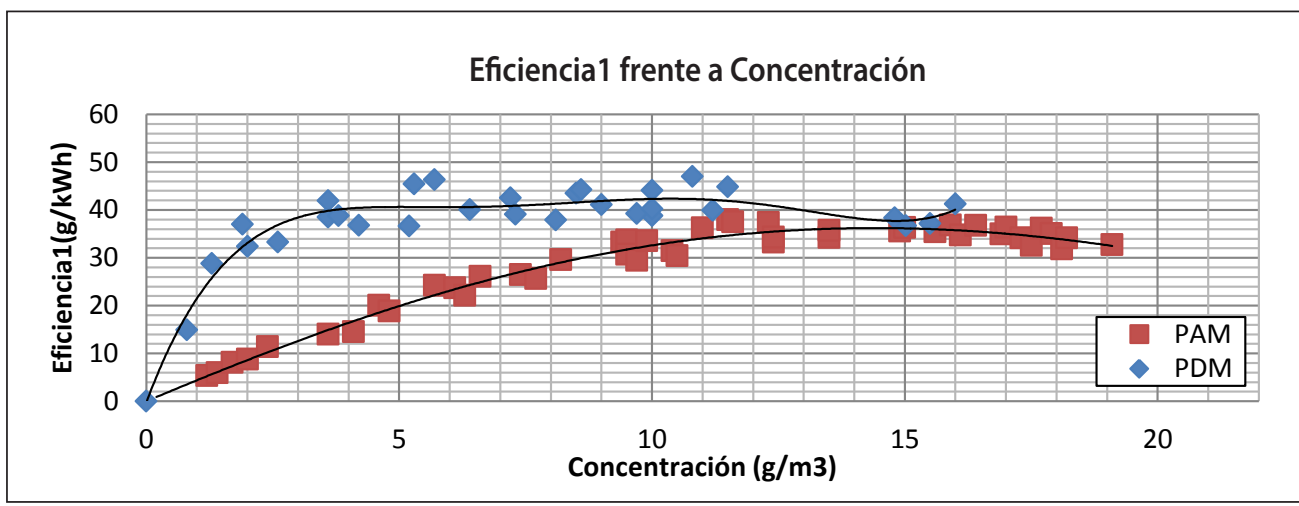

Figura 7. Eficiencia $\eta 1$ frente a concentración de $\mathrm{O}_{3}$ para la modulación PAM y PDM

Fuente: elaboración propia

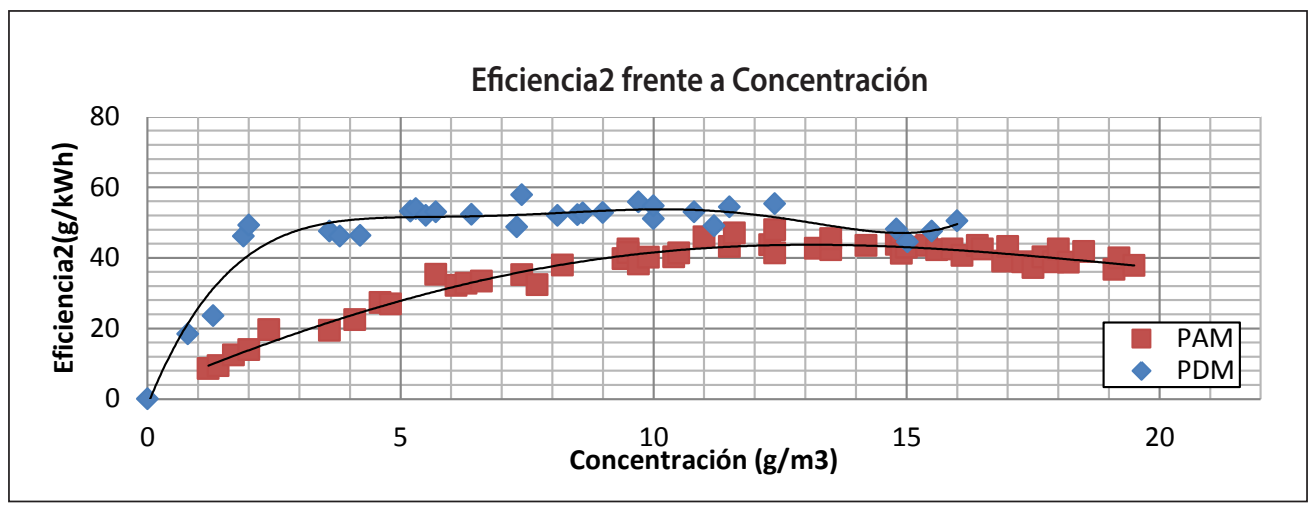

Figura 8. Eficiencia $\eta 2$ frente a concentración de 03 para la modulación PAM y PDM

Fuente: elaboración propia

Los resultados anteriores muestran para el caso de PAM que existen un conjunto de puntos en los cuales la eficiencia es alta. Estos corresponden a valores de concentración de ozono mayores a $12 \mathrm{~g} / \mathrm{m}^{3}$; de igual manera se observa que para concentraciones mayores a $16 \mathrm{~g} / \mathrm{m}^{3}$ la eficiencia decae; este efecto puede ser causado por calentamiento de la celda y a que no se opera a un campo eléctrico reducido óptimo [7]. Para el caso de PDM se presentó un mejoramiento de la eficiencia respecto a PAM en el rango entre 0 y $16 \mathrm{~g} / \mathrm{m}^{3}$ el cual corresponde a un mejoramiento del $60 \%$ del rango analizado; este mejoramiento se atribuye a la aplicación de una amplitud de voltaje óptima en los pulsos durante el tiempo $\mathrm{T}_{\mathrm{ON}}$ de la técnica PDM. Sin embargo, en el rango de concentraciones entre 0 y $2 \mathrm{~g} / \mathrm{m}^{3}$ la eficiencia es relativamente baja, lo cual puede estar relacionado con que los tiempos $\mathrm{T}_{\mathrm{ON}}$ de la modulación PDM son muy cortos $\mathrm{y}$ no permiten una descarga estable. 
Por otra parte, las curvas de eficiencia $\eta 1$ y $\eta 2$ tanto para PAM como PDM se igualan a partir de 15,5 y $16 \mathrm{~g} / \mathrm{kWh}$, punto en el cual el ciclo de trabajo en PDM es del $100 \%$. También se evidencia que la $\eta 1$ y la $\eta 2$ presentan un desempeño proporcional punto a punto en el rango medido. Con la técnica de modulación PDM se consigue un mejoramiento de la eficiencia en el rango de baja concentración y también se obtienen valores de máxima eficiencia superiores al obtenido por el método tradicional de modulación PAM, lo que se debe a la aplicación de la técnica PDM con pulsos fijados a una amplitud óptima.

En la Tabla 2 Resumen del desempeño de las técnicas de modulación evaluadas se resumen los valores de las características planteadas para las modulaciones PAM y PDM.

Tabla 2 Resumen del desempeño de las técnicas de modulación evaluadas

\begin{tabular}{|l|c|c|}
\hline \multicolumn{1}{|c|}{ Característica } & PAM & PDM \\
\hline$\eta 1$ máxima $(\mathrm{g} / \mathrm{kWh})$ & 39 & 45 \\
\hline$\eta 2$ máxima $(\mathrm{g} / \mathrm{kWh})$ & 47 & 56 \\
\hline Rango de [] $\mathrm{O}_{3}$ con $\eta 1$ Máxima $\left(\mathrm{g} / \mathrm{m}^{3}\right)$ & $12-16$ & $9-12$ \\
\hline Rango de [] $\mathrm{O}_{3}$ con $\eta 2$ Máxima $\left(\mathrm{g} / \mathrm{m}^{3}\right)$ & $12-16$ & $9-11$ \\
\hline Rango de [] $\mathrm{O}_{3}$ con mejora de $\eta 1$ respecto a PAM en $\left(\mathrm{g} / \mathrm{m}^{3}\right)$ & - & $0-15$ \\
\hline Rango de [] $\mathrm{O}_{3}$ con mejora de $\eta 2$ respecto a PAM en $\left(\mathrm{g} / \mathrm{m}^{3}\right)$ & - & $0-15$ \\
\hline
\end{tabular}

Fuente: elaboración propia

\section{CONCLUSIONES}

Se implementó la técnica de modulación de potencia PDM, fijando la amplitud de voltaje a un valor óptimo, el cual correspondería a un campo eléctrico óptimo. Este voltaje óptimo se determina de la curva característica de eficiencia de la celda de descarga DBD. Al aplicar la técnica PDM se observó un aumento de la eficiencia en todos los puntos de operación del sistema; no obstante, su efecto es más significativo en la zona de baja concentración de la curva característica. Por tanto, es posible incrementar la eficiencia de un sistema de generación de ozono, en el rango de puntos de operación de baja concentración mediante la implementación de la estrategia PDM. Sin embargo, no es posible aumentar la eficiencia con PDM mas allá de los límites físicos impuestos por la celda DBD. 


\section{AGRADECIMIENTOS}

Esta investigación hizo parte del proyecto Sustainable Biofuels for North Andean Countries desarrollado por los grupos de investigación GRALTA y GAOX. Se agradece a la Universidad del Valle y a la Dirección para el Desarrollo y la Cooperación del Gobierno Suizo (DDC) por su financiación.

\section{REFERENCIAS}

[1] D. F. Echeverry Ibarra, D. A. Cardona, A. Galvis, G. Aponte Mayor, and M. I. Gonzalez, "Evaluación del funcionamiento de un Generador de Ozono a escala piloto en la desinfección de agua para consumo humano", Ing. Y Compet., vol. 7, n. ${ }^{\circ}$ 1, pp. 65-72, 2005.

[2] F. Machuca Martínez, N. Caicedo, D. F. Echeverry Ibarra, and Y. Muñoz, "Treatment of crude vinasse from cane industry by ozone", in The 16th International Conference on Advanced Oxidation Technologies for Treatment of Water, Air and Soil, 2010.

[3] D. F. Echeverry Ibarra, H. Cadavid Ramírez, J. M. Alonso, G. Aponte Mayor, and A. Gálvis Castaño, "Experimental Results of a Cost-Effective Ozone Generator for Water Treatment in Colombia”, Ozone Sci. Eng., vol. 30, n. 3, pp. 202-209, May 2008.

[4] V. Gibalov and G. Pietsch, "On the Performance of Ozone Generators Working with Dielectric Barrier Discharges”, Ozone Sci. Eng., vol. 28, n.․ 2, pp. 119-124, May 2006.

[5] Y. Konishi, S. Wang, M. Ishibashi, and M. Nakaoka, "Series inductor compensated load resonant inverter type ozonizer with pulse density modulation control scheme and its evaluations", ISIE '97 Proceeding IEEE Int. Symp. Ind. Electron., vol. 2, pp. 347-352, 1997.

[6] M. T. Tsai and C. L. Chu, "Power control strategies evaluation of a series resonant inverter for atmosphere plasma applications”, 2009 IEEE Int. Symp. Ind. Electron., no. ISIE, pp. 632-637, Jul. 2009.

[7] V. I. Gibalov and G. J. Pietsch, "On the Performance of Ozone Generators Working with Dielectric Barrier Discharges,” Ozone Sci. Eng., vol. 28, n. 2, pp. 119-124, May 2006.

[8] I. D. Chalmers, L. Zanella, and S. J. MacGregor, "Ozone synthesis in oxygen in a dielectric barrier free configuration", in Digest of Technical Papers. Tenth IEEE International Pulsed Power Conference, 1995, vol. 2, pp. 1249-1254.

[9] W. J. M. Samaranayake, Y. Miyahara, T. Namihira, S. Katsuki, R. Hackam, and H. Akiyama, "Pulsed power production of ozone in $\mathrm{O} / \mathrm{sub} 2 / / \mathrm{N} / \mathrm{sub} 2 /$ in a coaxial reactor without dielectric layer," IEEE Trans. Dielectr. Electr. Insul., vol. 8, n. ${ }^{\circ}$ 5, pp. 826-831, 2001.

[10] T. Namihira, K. Shinozaki, S. Katsuki, R. Hackam, H. Akiyama, and T. Sakugawa, "Characteristics of ozonizer using pulsed power", in PPPS-2001 Pulsed Power Plasma Science 2001. 28th IEEE International Conference on Plasma Science and 13th IEEE International Pulsed Power Conference. Digest of Papers (Cat. No.01CH37251), vol. 2, pp. 1090-1093. 
[11] J. M. Alonso, J. García, A. J. Calleja, J. Ribas, and J. Cardesin, “Analysis, Design, and Experimentation of a High-Voltage Power Supply for Ozone Generation Based on Current-Fed Parallel-Resonant Push-Pull Inverter", IEEE Trans. Ind. Appl., vol. 41, n. ${ }^{\circ}$ 5, pp. 1364-1372, Sep. 2005.

[12] J. M. Alonso, J. Cardesin, J. a. Martín-Ramos, J. García, and M. Rico-Secades, “Using currentfed parallel-resonant inverters for electro-discharge applications: a case of study", Ninet. Annu. IEEE Appl. Power Electron. Conf. Expo. 2004. APEC '04., vol. 00, n. ${ }^{\circ}$ C, pp. 10-115, 2004.

[13] D. F. Echeverry Ibarra, "Aumento de la eficiencia del sistema de descarga de barrera dieléctrica aplicado en la generación de ozono”, Universidad del Valle, 2008.

[14] R. Díaz, A. Márquez, D. Menéndez, and R. Pérez, "Medium frequency pulse train ozone generation", Ozone Sci. Eng., vol. 21, n. ${ }^{\circ}$ 6, pp. 635-642, 1999.

[15] S. Masuda, K. Akutsu, M. Kuroda, Y. Awatsu, and Y. Shibuya, "A ceramic-based ozonizer using high-frequency discharge,” IEEE Trans. Ind. Appl., vol. 24, n. 2, pp. 223-231, 1988.

[16] T. Cieplak, C. Yamabe, S. Ihara, S. Satoh, J. Pawlat, J. Cieplak, and I. Polio, “Ozone Generation Using Plate Rotating Electrode Ozonizer- Effect Of Electrode Rotation And Discharge Analysis Method," Ozone Sci. Eng., vol. 22, n. ${ }^{\circ}$ 6, pp. 563-574, Dec. 2000.

[17] A. Lukanin and O. Khasanov, "Development of small and efficient ozone generators using corona discharge", in 5th Korea-Russia International Symposium on Science and Technology. Proceedings. KORUS 2001 (Cat. No.01EX478), vol. 1, pp. 282-84.

[18] J. Suehiro, M. Takahashi, Y. Nishi, W. Ding, K. Imasaka, and M. Hara, "Improvement of the Ozone Generation Efficiency by Silent Discharge at Cryogenic Temperature", IEEJ Trans. Fundam. Mater., vol. 124, n. ${ }^{\circ}$, pp. 791-796, 2004.

[19] M. M. Peretz, S. Ben-yaakov, and P. Box, "Analysis of the Current-Fed Push-Pull Parallel Resonant Inverter Implemented with Unidirectional Switches", in Power Electronics Specialists Conference PESC'05, 2005, pp. 880-884.

[20] C. Ordiz, J. M. Alonso, M. a. Dalla Costa, J. Ribas, and a. J. Calleja, “Development of a highvoltage closed-loop power supply for ozone generation," 2008 Twenty-Third Annu. IEEE Appl. Power Electron. Conf. Expo., pp. 1861-867, Feb. 2008. 